\title{
A macroeconomic mathematical model for the national income of a union of countries with interaction and trade
}

\author{
loannis K. Dassios ${ }^{1 *}$ and Mel T. Devine ${ }^{1,2,3}$
}

*Correspondence:
jdasios@math.uoa.gr
${ }^{1}$ Mathematics Applications
Consortium for Science
and Industry (MACSI),
Department of Mathematics
and Statistics, University
of Limerick, Limerick, Ireland
Full list of author information
is available at the end of the
article

${ }^{*}$ Correspondence: jdasios@math.uoa.gr Mathematics Application Consortium for Science Department of Mathematics and Statistics, University article (1) CrossMark

\begin{abstract}
In this article, we assume a union of countries where each national economy interacts with the others. We propose a new model where (a) delayed variables are incorporated into the system of equations and (b) the interaction element is restricted into the annual governmental expenditure that is determined according to the experience of the total system and the trade relations of these countries (exports-imports). In addition, we consider the equilibrium(s) of the model (a discrete-time system) and study properties for stability, the appropriate control actions as well as the total system design in order to obtain a stable situation. Finally, a practical application is also investigated that provides further insight and better understanding as regards the system design and produced business cycles.

Keywords: National economy, Network, Difference equations, Stability, Control, Trade
\end{abstract}

\section{Background}

Keynesian macroeconomics inspired the seminal work of Samuelson (1939), who introduced the business cycle theory. Although primitive and using only the demand point of view, the Samuelson's prospect still provides an excellent insight into the problem and justification of business cycles appearing in national economies. In the past decades, many more sophisticated models have been proposed by other researchers (Chari 1994; Chow 1985; Dalla and Varelas 2015; Dalla et al. 2016; Dassios et al. 2014; Dassios and Kalogeropoulos 2014; Dassios and Zimbidis 2014; Day 1999; Karpetis and Varelas 2012; Kotsios and Leventidis 2004; Kotsios and Kostarakos 2015, 2016; Machado et al. 2015; Matsumoto and Szidarovszky 2015; Puu et al. 2004; Rosser 2000; Westerhoff 2006; Wincoop 1996). All these models use superior and more delicate mechanisms involving monetary aspects, inventory issues, business expectation, borrowing constraints, welfare gains and multi-country consumption correlations.

Some of the previous articles also contribute to the discussion for the inadequacies of Samuelson's model. The basic shortcoming of the original model is: the incapability to produce a stable path for the national income when realistic values for the different parameters (multiplier and accelerator parameters) are entered into the system of 
equations. Of course, this statement contradicts with the empirical evidence which supports temporary or long-lasting business cycles.

Business cycle models are important as they are used to investigate economic behaviour in the study of optimal fiscal and monetary policies as well as examine the effects of economic shocks (Chari 1994). In addition, such models are also used to study investment, consumption and inventory cycles (Dalla and Varelas 2015; Dalla et al. 2016). The more realistic a business cycle model is, the more informed the analysis it provides. The model described in the present work is highly realistic as it incorporates multiple delays and trade factors for union of unlimited countries for an unlimited number of years and thus incorporates more of the factors that determine national incomes than any of the previous works seen in the literature. It also succeeds to provide a comprehensive explanation for the emergence of business cycles, while it also produces a stable trajectory for the expectation of the national income of each country which is part of the network.

The rest of this paper is organised as follows. In Sect. 2, we propose a new model for a national economy into a multi-country context where the interaction element is restricted to the annual governmental expenditure, which is determined according to the experience of the total system, and the trade relations of these countries (exportsimports). In addition, delayed variables are incorporated into the system of equations based on delayed information. Section 3 investigates the stability of the equilibrium state of the system and suggests a typical state feedback action for the different parameters involved, while it also proposes how to design the corresponding solution trajectories. Section 4 contains a practical application that provides further insight and better understanding as regards the control actions, system design and produced business cycles. Sections 5 and 6 conclude the entire paper.

\section{Methods}

The proposed model for a network of $i$ countries, $i=2,3, \ldots, \omega$, is based on the following assumptions:

Assumption 1 National income $T_{k}^{i}$ for the $i$ country in year $k$ equals to the summation of the following elements: consumption, $C_{k}^{i}$, private investment, $I_{k}^{i}$, governmental expenditure $G_{k}^{i}$ and exports $X_{k}^{i}$ less imports $Y_{k}^{i}$

$$
T_{k}^{i}=C_{k}^{i}+I_{k}^{i}+G_{k}^{i}+X_{k}^{i}-Y_{k}^{i}
$$

Assumption 2 Consumption $C_{k}^{i}$ in year $k$ depends on past income (on more than one past year's value) and on marginal tendencies to consume, modelled with $a_{1}^{i}, a_{2}^{i}, \ldots, a_{n}^{i}$, the multiplier parameters, where $0<a_{1}^{i}+a_{2}^{i}+\cdots+a_{n}^{i}<1$

$$
C_{k}^{i}=a_{1}^{i} T_{k-1}^{i}+a_{2}^{i} T_{k-2}^{i}+\cdots+a_{n}^{i} T_{k-n}^{i} .
$$

Assumption 3 Private investment $I_{k}^{i}$ in year $k$ depends on consumption changes and on the positive accelerator factors $b_{1}^{i}, b_{2}^{i}, \ldots, b_{m}^{i}$. Consequently, $I_{k}^{i}$ depends on the respective national income changes,

$$
I_{k}^{i}=b_{1}^{i}\left(C_{k}^{i}-C_{k-1}^{i}\right)+b_{2}^{i}\left(C_{k-1}^{i}-C_{k-2}^{i}\right)+\cdots+b_{m}^{i}\left(C_{k-m+1}^{i}-C_{k-m}^{i}\right) .
$$


By using (2) we get

$$
I_{k}^{i}=b_{1}^{i}\left(\sum_{q=1}^{n} a_{q}^{i} T_{k-q}^{i}-\sum_{q=1}^{n} a_{q}^{i} T_{k-(q+1)}^{i}\right)+\cdots+b_{m}^{i}\left(\sum_{q=1}^{n} a_{q}^{i} T_{k-(q+m-1)}^{i}-\sum_{q=1}^{n} a_{q}^{i} T_{k-(q+m)}^{i}\right),
$$

or, equivalently,

$$
I_{k}^{i}=\sum_{j=1}^{m} b_{j}^{i}\left(\sum_{q=1}^{n} a_{q}^{i} T_{k-(q+j-1)}^{i}-\sum_{q=1}^{n} a_{q}^{i} T_{k-(q+j)}^{i}\right) .
$$

Assumption 4 The governmental expenditure depends not only on the country's national income but also on the national income of the other countries participating into the multi-country union, i.e. governmental expenditure $G_{k}^{i}$ in year $k$ obeys the feedback law $I_{k}^{i}$ in year $k$ and depends on consumption changes and on the positive accelerator factors $c_{1}^{i, d}, c_{2}^{i, d}, \ldots, c_{p}^{i, d}$. Consequently, $G_{k}^{i}$ is equal to,

$$
G_{k}^{i}=\bar{G}^{i}+\sum_{d=1}^{\omega}\left[c_{1}^{i, d} T_{k-1}^{d}+c_{2}^{i, d} T_{k-2}^{d}+\cdots+c_{p}^{i, d} T_{k-p}^{d}\right]
$$

Note the $G_{k}^{i}$, may be a fully controlled item by the governments, i.e.

$$
G_{k}^{i}=\bar{G}_{k}^{i}+\sum_{d=1}^{\omega}\left[c_{1}^{i, d} T_{k-1}^{d}+c_{2}^{i, d} T_{k-2}^{d}+\cdots+c_{p}^{i, d} T_{k-p}^{d}\right] .
$$

Assumption 5 Imports of country $i$ in year $k$ depend on past income and on the multipliers $m_{t}^{i}$ :

$$
Y_{k}^{i}=m_{1}^{i} T_{k-1}^{i}+m_{2}^{i} T_{k-2}^{i}+\cdots+m_{t}^{i} T_{k-t}^{i}
$$

or, equivalently,

$$
Y_{k}^{i}=\sum_{u=1}^{t} m_{u}^{i} T_{k-u}^{i}
$$

Assumption 6 The imports are also a linear sum of the exports of all other countries in year $k$ :

$$
Y_{k}^{i}=\bar{e}_{i, 1} X_{k}^{1}+\bar{e}_{i, 2} X_{k}^{2}+\cdots+\bar{e}_{i, \omega} X_{k}^{\omega},
$$

or, equivalently,

$$
Y_{k}^{i}=\sum_{q=1}^{\omega} \bar{e}_{i, q} X_{k}^{q} .
$$


Now let $\bar{Y}_{k}, \bar{E}$ and $\bar{X}_{k}$ be $\omega \times 1, \omega \times \omega$ and $\omega \times 1$ matrices, respectively. The $i$ th elements of $\bar{Y}_{k}$ and $\bar{X}_{k}$ are $Y_{k}^{i}$ and $X_{k}^{i}$, respectively, while $\bar{e}_{i, q}$ is the entry in the $i$ th row and $q$ th column of $\bar{E}$. As a result, the above expression can be written as

$$
\bar{Y}_{k}=\bar{E} \bar{X}_{k},
$$

Let

$$
E=\left\{\begin{array}{ll}
\bar{E}^{-1}, & \text { if } \operatorname{det} \bar{E} \neq 0 \\
\bar{E}^{\dagger}, & \text { if } \operatorname{det} \bar{E}=0
\end{array}\right\}
$$

Then

$$
\bar{X}_{k}=E \bar{Y}_{k},
$$

which means the $i$ th row of $\bar{X}_{k}$ may be written as

$$
X_{k}^{i}=e_{i, 1} Y_{k}^{1}+e_{i, 2} Y_{k}^{2}+\cdots+e_{i, \omega} Y_{k}^{\omega},
$$

or, equivalently,

$$
X_{k}^{i}=\sum_{q=1}^{\omega} e_{i, q} Y_{k}^{q},
$$

where $e_{i, d}$ is the entry in the $i$ th row and $q$ th column of $E$. We also assume

$$
\begin{array}{ll}
\sum_{q=1}^{\omega} e_{i, q}=1, & \forall i=1,2, \ldots, \omega, \\
e_{i, i}=0, & \forall i=1,2, \ldots, \omega .
\end{array}
$$

Combining equations (6) and (5) gives

$$
X_{k}^{i}=\sum_{q=1}^{\omega}\left[e_{i, q} \sum_{u=1}^{t} m_{u}^{q} T_{k-u}^{q}\right] .
$$

Hence, by replacing (2), (3), (4), (5) and (7) into (1), the national income is determined via the following high-order linear difference equation

$$
\begin{aligned}
T_{k}^{i}= & \bar{G}^{i}+\sum_{q=1}^{p} \sum_{d=1}^{\omega} c_{q}^{i, d} T_{k-q}^{d}+\sum_{q=1}^{n} a_{q}^{i} T_{k-q}^{i}+\sum_{j=1}^{m} b_{j}^{i}\left(\sum_{q=1}^{n} a_{q}^{i} T_{k-(q+j-1)}^{i}-\sum_{q=1}^{n} a_{q}^{i} T_{k-(q+j)}^{i}\right) \\
& +\sum_{q=1}^{\omega}\left[e_{i, q} \sum_{u=1}^{t} m_{u}^{q} T_{k-u}^{q}\right]-\sum_{u=1}^{t} m_{u}^{i} T_{k-u}^{i}, \quad i=1,2, \ldots, \omega
\end{aligned}
$$

Although Eq. (8) describes the general case of our model, its form is quite difficult and probably not so informative for further studies. Hence, we restrict our attention to a more realistic case where consumption depends on $p-1$ past year's income values, private investments depend on consumption changes within the last year, and governmental expenditures and export-imports depend on $p$ past year's income values. Then, (8) takes the form 


$$
\begin{aligned}
T_{k}^{i}= & \bar{G}^{i}+\sum_{q=1}^{p} \sum_{d=1}^{\omega} c_{q}^{i, d} T_{k-q}^{d}+\sum_{q=1}^{p-1} a_{q}^{i} T_{k-q}^{i}+\sum_{q=1}^{p-1} b_{1}^{i} a_{q}^{i}\left(T_{k-q}^{i}-T_{k-(q+1)}^{i}\right) \\
& +\sum_{d=1}^{\omega}\left[e_{i, d} \sum_{q=1}^{p} m_{q}^{d} T_{k-q}^{d}\right]-\sum_{q=1}^{p} m_{q}^{i} T_{k-q}^{i}, \quad i=1,2, \ldots, \omega .
\end{aligned}
$$

\section{Results}

In this section, we study the stability of the equilibrium state of (9). In addition, by using concepts and results of linear control theory for time-invariant linear discrete state equations (Azzo and Houpis 1995; Dorf 1983; Kuo 1996; Ogata 1987), we investigate the case in which the governmental expenditure $\bar{G}_{k}^{i}$ of country $i$ at time $k, i=1,2, \ldots, \omega$, is a fully controlled variable, as also mentioned in Assumption 4 in Sect. 2. We prove the following theorem.

Theorem 3.1 Assume a union of $\omega$ countries and let $T_{k}^{i}, i=1,2, \ldots, \omega$, be the national income for the ith country in year $k$. If for every country that participates into the multicountry union, consumption depends on $p-1$ past year's income values, private investments depend on consumption changes within the last year, and governmental expenditures and export-imports depend on p past year's income values, then:

1. The national income is determined via the following linear matrix difference equation

$$
\begin{aligned}
T_{k}= & \bar{G}+\left[C^{(1)}+A^{(1)}+B A^{(1)}+\left(E-I_{\omega}\right) M^{(1)}\right] T_{k-1} \\
& +\sum_{q=2}^{p-1}\left\{\left[C^{(q)}+A^{(q)}+B\left(A^{(q)}-A^{(q-1)}\right)+\left(E-I_{\omega}\right) M^{(q)}\right] T_{k-q}\right\} \\
& +\left[C^{(p)}-B A^{(p-1)}+\left(E-I_{\omega}\right) M^{(p)}\right] T_{k-p}, \quad k \geq 0, \quad p \geq 3 .
\end{aligned}
$$

where $I_{\omega}$ is the identity matrix and

$$
\begin{aligned}
T_{k} & =\left[T_{k}^{i}\right]_{i=1,2, \ldots, \omega}, \\
A^{(q)} & =\operatorname{diag}\left\{a_{q}^{i}\right\}_{i=1,2, \ldots, \omega}, \quad q=1,2, \ldots, p-1, \\
B & =\operatorname{diag}\left\{b^{i}\right\}_{i=1,2, \ldots, \omega}, \\
C^{(q)} & =\left[c_{q}^{i, d}\right]_{1 \leq i, d \leq \omega}, \quad q=1,2, \ldots, p, \\
E & =\left[e_{i, d}\right]_{1 \leq i, d \leq \omega}, \\
M^{(q)} & =\operatorname{diag}\left\{m_{q}^{i}\right\}_{i=1,2, \ldots, \omega}, \quad q=1,2, \ldots, p, \\
G & =\left[\bar{G}^{i}\right]_{i=1,2, \ldots, \omega} .
\end{aligned}
$$

The parameters $a_{q}^{i}, b^{i}, c_{q}^{i, d}, e_{i, d}, m_{q}^{i}$, are defined in (2), (3), (4), (5) and (6). In addition, if $\operatorname{det}\left[\sum_{q=1}^{p-1}\left\{C^{(q)}+A^{(q)}+\left(E-I_{\omega}\right) M^{(q)}\right\}+C^{(p)}+\left(E-I_{\omega}\right) M^{(p)}+B A^{(1)}\right] \neq 0$,

then the equilibrium 


$$
T^{*}=\left[\sum_{q=1}^{p-1}\left\{C^{(q)}+A^{(q)}+\left(E-I_{\omega}\right) M^{(q)}\right\}+C^{(p)}+\left(E-I_{\omega}\right) M^{(p)}+B A^{(1)}\right]^{-1} G
$$

of (10) is asymptotically stable, i.e. $\lim _{k \rightarrow \infty} T_{k}=T^{*}$, if and only if $\forall i, i=1,2, \ldots, \omega$ and $\phi(\lambda)=0$ :

$|\lambda|<1, \quad \lambda \in \mathbb{C}$.

where

$$
\begin{aligned}
\phi(\lambda)= & \operatorname{det}\left(-\lambda^{p}+\left[C^{(1)}+A^{(1)}+B A^{(1)}+\left(E-I_{\omega}\right) M^{(1)}\right] \lambda^{p-1}\right. \\
& +\sum_{q=2}^{p-1}\left\{\left[C^{(q)}+A^{(q)}+B\left(A^{(q)}-A^{(q-1)}\right)+\left(E-I_{\omega}\right) M^{(q)}\right] \lambda^{p-q}\right\} \\
& \left.+\left[C^{(p)}-B A^{(p-1)}+\left(E-I_{\omega}\right) M^{(p)}\right]\right) .
\end{aligned}
$$

2. If governmental expenditure is a fully controlled variable for each country, then the national income is determined via the following system

$Y_{k+1}=F Y_{k}+Q G_{k}$.

where $Y_{k}=\left[Y_{k}^{i}\right]_{i=1,2, \ldots, \omega} \in \mathbb{R}^{p \omega \times 1}, F \in \mathbb{R}^{p \omega \times p \omega}, G_{k}=\left[G_{k}^{i}\right]_{i=1,2, \ldots, \omega} \in \mathbb{R}^{\omega \times 1}$ and

$$
F=\left[\begin{array}{llllll}
0_{\omega, \omega} & I_{\omega} & 0_{\omega, \omega} & \cdots & 0_{\omega, \omega} & 0_{\omega, \omega} \\
0_{\omega, \omega} & 0_{\omega, \omega} & I_{\omega} & \cdots & 0_{\omega, \omega} & 0_{\omega, \omega} \\
\vdots & \vdots & \vdots & \ddots & \vdots & \vdots \\
0_{\omega, \omega} & 0_{\omega, \omega} & 0_{\omega, \omega} & \cdots & 0_{\omega, \omega} & I_{\omega} \\
v_{1} & v_{2} & v_{3} & \cdots & v_{p-1} & v_{p}
\end{array}\right], \quad Q=\left[\begin{array}{c}
0_{\omega, \omega} \\
0_{\omega, \omega} \\
\vdots \\
0_{\omega, \omega} \\
I_{\omega}
\end{array}\right] \in \mathbb{R}^{p \omega \times \omega} .
$$

where

$$
\begin{aligned}
v_{1}= & C^{(p)}-B A^{(p-1)}+\left(E-I_{\omega}\right) M^{(p)} \\
v_{2}= & C^{(p-1)}+A^{(p-1)}+B\left(A^{(p-1)}-A^{(p-2)}\right)+\left(E-I_{\omega}\right) M^{(p-1)} \\
v_{3}= & C^{(p-2)}+A^{(p-2)}+B\left(A^{(p-2)}-A^{(p-3)}\right)+\left(E-I_{\omega}\right) M^{(p-2)} \\
\vdots & \\
v_{p-1}= & C^{(2)}+A^{(2)}+B\left(A^{(2)}-A^{(1)}\right)+\left(E-I_{\omega}\right) M^{(2)} \\
v_{p}= & C^{(1)}+A^{(1)}+B A^{(1)}+\left(E-I_{\omega}\right) M^{(1)}
\end{aligned}
$$

In addition, there exists a state feedback law of the form

$G_{k}=-K Y_{k}$,

where $K=\left[\begin{array}{llll}K_{1} & K_{2} & \cdots & K_{p}\end{array}\right] \in \mathbb{C}^{\omega \times p \omega}, K_{i} \in \mathbb{C}^{\omega \times \omega}$, such that the eigenvalues $\tilde{\lambda}$ of the closed-loop system can be assigned arbitrarily and be given as the roots of the polyno$\operatorname{mial} \tilde{\phi}(\tilde{\lambda})$ :

$$
\begin{aligned}
\tilde{\phi}(\tilde{\lambda})= & \operatorname{det}\left(-\tilde{\lambda}^{p}+\left[C^{(1)}+A^{(1)}+B A^{(1)}+\left(E-I_{\omega}\right) M^{(1)}-K_{p}\right] \tilde{\lambda}^{p-1}\right. \\
& +\sum_{q=2}^{p-1}\left\{\left[C^{(q)}+A^{(q)}+B\left(A^{(q)}-A^{(q-1)}\right)+\left(E-I_{\omega}\right) M^{(q)}-K_{p-q}\right] \tilde{\lambda}^{p-q}\right\} \\
& \left.+\left[C^{(p)}-B A^{(p-1)}+\left(E-I_{\omega}\right) M^{(p)}-K_{1}\right]\right) .
\end{aligned}
$$


Proof For the proof of (a), from (9), $\forall i=1,2, \ldots, \omega$ :

$$
\begin{aligned}
T_{k}^{i}= & \bar{G}^{i}+\sum_{q=1}^{p} \sum_{d=1}^{\omega} c_{q}^{i, d} T_{k-q}^{d}+\sum_{q=1}^{p-1} a_{q}^{i} T_{k-q}^{i}+\sum_{q=1}^{p-1} b_{1}^{i} a_{q}^{i}\left(T_{k-q}^{i}-T_{k-(q+1)}^{i}\right) \\
& +\sum_{d=1}^{\omega}\left[e_{i, d} \sum_{q=1}^{p} m_{q}^{d} T_{k-q}^{d}\right]-\sum_{q=1}^{p} m_{q}^{i} T_{k-q}^{i},
\end{aligned}
$$

or, equivalently,

$$
\begin{aligned}
T_{k}^{i}= & \bar{G}^{i}+\sum_{q=1}^{p}\left[\begin{array}{llll}
c_{q}^{i, 1} & c_{q}^{i, 2} & \cdots & c_{q}^{i, \omega}
\end{array}\right]\left[\begin{array}{c}
T_{k-q}^{1} \\
T_{k-q}^{2} \\
\cdots \\
T_{k-q}^{\omega}
\end{array}\right]+\sum_{q=1}^{p-1} a_{q}^{i} T_{k-q}^{i}+\sum_{q=1}^{p-1} b_{1}^{i} a_{q}^{i}\left(T_{k-q}^{i}-T_{k-(q+1)}^{i}\right) \\
& +\left[\begin{array}{llll}
e_{i, 1} & e_{i, 2} & \cdots & e_{i, \omega}
\end{array}\right]\left[\begin{array}{c}
\sum_{q=1}^{p} m_{q}^{1} T_{k-q}^{1} \\
\sum_{q=1}^{p} m_{q}^{2} T_{k-q}^{2} \\
\cdots \\
\sum_{q=1}^{p} m_{q}^{\omega} T_{k-q}^{\omega}
\end{array}\right]-\sum_{q=1}^{p} m_{q}^{i} T_{k-q}^{i},
\end{aligned}
$$

or, equivalently,

$$
\begin{aligned}
T_{k}^{i}= & \bar{G}^{i}+\sum_{q=1}^{p}\left[\begin{array}{llll}
c_{q}^{i, 1} & c_{q}^{i, 2} \cdots & c_{q}^{i, \omega}
\end{array}\right]\left[\begin{array}{c}
T_{k-q}^{1} \\
T_{k-q}^{2} \\
\cdots \\
T_{k-q}^{\omega}
\end{array}\right] \\
& +\sum_{q=1}^{p-1} a_{q}^{i} T_{k-q}^{i}+\sum_{q=1}^{p-1} b_{1}^{i} a_{q}^{i} T_{k-q}^{i}-\sum_{q=2}^{p} b_{1}^{i} a_{q-1}^{i} T_{k-q}^{i} \\
& +\sum_{q=1}^{p}\left[\begin{array}{llll}
e_{i, 1} & e_{i, 2} & \cdots & e_{i, \omega}
\end{array}\right] \operatorname{diag}\left\{m_{q}^{i}\right\}_{i=1,2, \ldots, \omega}\left[\begin{array}{c}
T_{k-q}^{1} \\
T_{k-q}^{2} \\
\cdots \\
T_{k-q}^{\omega}
\end{array}\right]-\sum_{q=1}^{p} m_{q}^{i} T_{k-q}^{i},
\end{aligned}
$$

or, equivalently,

$$
\begin{aligned}
T_{k}^{i}= & \bar{G}^{i}+\sum_{q=1}^{p}\left\{\left(\left[\begin{array}{lll}
c_{q}^{i, 1} & \cdots & c_{q}^{i, \omega}
\end{array}\right]+\left[\begin{array}{lll}
e_{i, 1} & \cdots & e_{i, \omega}
\end{array}\right] \operatorname{diag}\left\{m_{q}^{i}\right\}_{i=1, \ldots, \omega}\right)\left[\begin{array}{c}
T_{k-q}^{1} \\
\cdots \\
T_{k-q}^{\omega}
\end{array}\right]-m_{q}^{i} T_{k-q}^{i}\right\} \\
& +\sum_{q=1}^{p-1}\left\{a_{q}^{i} T_{k-q}^{i}+b_{1}^{i} a_{q}^{i} T_{k-q}^{i}\right\}-\sum_{q=2}^{p} b_{1}^{i} a_{q-1}^{i} T_{k-q}^{i},
\end{aligned}
$$

or, equivalently, by denoting $b_{1}^{i}$ as $b^{i}$ 


$$
\begin{aligned}
& T_{k}^{i}=\bar{G}^{i}+\left(\left[\begin{array}{lll}
c_{1}^{i, 1} & \cdots & c_{1}^{i, \omega}
\end{array}\right]+\left[\begin{array}{lll}
e_{i, 1} & \cdots & e_{i, \omega}
\end{array}\right] \operatorname{diag}\left\{m_{1}^{i}\right\}_{i=1, \ldots, \omega}\right)\left[\begin{array}{c}
T_{k-1}^{1} \\
\cdots \\
T_{k-1}^{\omega}
\end{array}\right] \\
& +\left(a_{1}^{i}+b_{1}^{i} a_{1}^{i}-m_{1}^{i}\right) T_{k-1}^{i}+\sum_{q=2}^{p-1}\left\{\left(\left[\begin{array}{ccc}
c_{q}^{i, 1} \cdots & c_{q}^{i, \omega}
\end{array}\right]\right.\right. \\
& \left.+\left[\begin{array}{lll}
e_{i, 1} & \cdots & e_{i, \omega}
\end{array}\right] \operatorname{diag}\left\{m_{q}^{i}\right\}_{i=1, \ldots, \omega}\right)\left[\begin{array}{c}
T_{k-q}^{1} \\
\cdots \\
T_{k-q}^{\omega}
\end{array}\right] \\
& \left.+\left(a_{q}^{i}+b^{i}\left(a_{q}^{i}-a_{q-1}^{i}\right)-m_{q}^{i}\right) T_{k-q}^{i}\right\}+\left(\left[c_{p}^{i, 1} \cdots c_{p}^{i, \omega}\right]\right. \\
& \left.+\left[\begin{array}{lll}
e_{i, 1} & \cdots & e_{i, \omega}
\end{array}\right] \operatorname{diag}\left\{m_{p}^{i}\right\}_{i=1, \ldots, \omega}\right)\left[\begin{array}{c}
T_{k-p}^{1} \\
\cdots \\
T_{k-p}^{\omega}
\end{array}\right]+\left(b_{1}^{i} a_{p}^{i}-m_{p}^{i}\right) T_{k-p}^{i},
\end{aligned}
$$

or, equivalently, in matrix form,

$$
\begin{aligned}
T_{k}= & \bar{G}+\left(C^{(1)}+E M^{(1)}+A^{(1)}+B A^{(1)}-M^{(1)}\right) T_{k-1}^{i} \\
& +\sum_{q=2}^{p-1}\left\{\left(C^{(q)}+E M^{(q)}+A^{(q)}+B\left(A^{(q)}-A^{(q-1)}\right)-M^{(q)}\right) T_{k-q}\right\} \\
& +\left(C^{(p)}+E M^{(p)}+B A^{(p)}-M^{(p)}\right) T_{k-p}^{i},
\end{aligned}
$$

which leads to (10). Let $T^{*}$ be the equilibrium of (10). Then, for $\operatorname{det}\left[\sum_{q=1}^{p-1}\left\{C^{(q)}+A^{(q)}+\left(E-I_{\omega}\right) M^{(q)}\right\}+C^{(p)}+\left(E-I_{\omega}\right) M^{(p)}+B A^{(1)}\right] \neq 0$ we have

$$
\begin{aligned}
T^{*}= & \bar{G}+\left\{C^{(1)}+A^{(1)}+B A^{(1)}+\left(E-I_{\omega}\right) M^{(1)}\right. \\
& +\sum_{q=2}^{p-1}\left[C^{(q)}+A^{(q)}+B\left(A^{(q)}-A^{(q-1)}\right)+\left(E-I_{\omega}\right) M^{(q)}\right] \\
& \left.+C^{(p)}-B A^{(p-1)}+\left(E-I_{\omega}\right) M^{(p)}\right\} T^{*}
\end{aligned}
$$

or, equivalently,

$$
T^{*}=\left[\sum_{q=1}^{p-1}\left\{C^{(q)}+A^{(q)}+\left(E-I_{\omega}\right) M^{(q)}\right\}+C^{(p)}+\left(E-I_{\omega}\right) M^{(p)}+B A^{(1)}\right]^{-1} \bar{G} .
$$

We are interested in the asymptotic stability of the equilibrium, i.e. when $\lim _{k \rightarrow \infty} T_{k}=T^{*}$. By adopting the notation

$$
\begin{gathered}
T_{k-p}=Y_{k}^{1}, \\
T_{k-p+1}=Y_{k}^{2}, \\
\vdots \\
T_{k-2}=Y_{k}^{p-1} \\
T_{k-1}=Y_{k}^{p}
\end{gathered}
$$

and 


$$
\begin{aligned}
& T_{k-p+1}=Y_{k+1}^{1}=Y_{k}^{2}, \\
& T_{k-p+2}=Y_{k+1}^{2}=Y_{k}^{3}, \\
& \quad \vdots \\
& T_{k-1}=Y_{k+1}^{p-1}=Y_{k}^{p} \\
& T_{k}=Y_{k+1}^{p}=\bar{G}+\left(C^{(1)}+A^{(1)}+B A^{(1)}+\left(E-I_{\omega}\right) M^{(1)}\right) Y_{k}^{p} \\
& \quad+\sum_{q=2}^{p-1}\left\{\left(C^{(q)}+A^{(q)}+B\left(A^{(q)}-A^{(q-1)}\right)+\left(E-I_{\omega}\right) M^{(q)}\right) Y_{k}^{q}\right\} \\
& \quad+\left(C^{(p)}-B A^{(p-1)}+\left(E-I_{\omega}\right) M^{(p)}\right) Y_{k}^{1} .
\end{aligned}
$$

Then, the matrix difference equation (10) takes the form

$$
Y_{k+1}=F Y_{k}+\left[\begin{array}{c}
0_{\omega, 1} \\
\vdots \\
0_{\omega, 1} \\
G
\end{array}\right] .
$$

The stability of the above system depends on the eigenvalues of $F$. Let $\lambda$ be an eigenvalue of $F$. Then, the equilibrium of (10) is asymptotic stable if and only if

$$
|\lambda|<1, \lambda \in \mathbb{C} .
$$

In addition, if $U=\left[\begin{array}{c}U_{1} \\ U_{2} \\ \vdots \\ U_{p}\end{array}\right]$ is an eigenvector of the eigenvalue $\lambda$, then

$$
\left[\begin{array}{cccccc}
0_{\omega, \omega} & I_{\omega} & 0_{\omega, \omega} & \cdots & 0_{\omega, \omega} & 0_{\omega, \omega} \\
0_{\omega, \omega} & 0_{\omega, \omega} & I_{\omega} & \cdots & 0_{\omega, \omega} & 0_{\omega, \omega} \\
\vdots & \vdots & \vdots & \ddots & \vdots & \vdots \\
0_{\omega, \omega} & 0_{\omega, \omega} & 0_{\omega, \omega} & \cdots & 0_{\omega, \omega} & I_{\omega} \\
v_{1} & v_{2} & v_{3} & \cdots & v_{p-1} & v_{p}
\end{array}\right] U=\lambda U
$$

or, equivalently,

$$
\begin{gathered}
U_{2}=\lambda U_{1}, \\
U_{3}=\lambda U_{2}, \\
\vdots \\
U_{p}=\lambda U_{p-1}, \\
\sum_{i=1}^{p} v_{i} U_{i}=\lambda U_{p},
\end{gathered}
$$

or, equivalently,

$$
\begin{gathered}
U_{2}=\lambda U_{1}, \\
U_{3}=\lambda^{2} U_{1}, \\
\vdots \\
U_{p}=\lambda^{p-1} U_{1}, \\
v_{1} U_{1}+v_{2} U_{2}+\cdots+v_{p} U_{p}=\lambda U_{p} .
\end{gathered}
$$


By replacing the first $p-1$ equations into the $p$ th equation of the above expression, we get

$$
v_{1} U_{1}+\lambda v_{2} U_{1}+\cdots+\lambda^{p-1} v_{p} U_{1}=\lambda^{p} U_{1}
$$

or, equivalently,

$$
\left[v_{1}+\lambda v_{2}+\cdots+\lambda^{p-1} v_{p}-\lambda^{p} I_{\omega}\right] U_{1}=0 .
$$

Hence, the eigenvalues $\lambda$ are the roots of the polynomial $\phi(\lambda)=\operatorname{det}\left[v_{1}+\lambda v_{2}+\cdots+\lambda^{p-1} v_{p}-\lambda^{p} I_{\omega}\right.$, which by replacing (13) is polynomial (11). Thus, the equilibrium $T^{*}$ of (10) is asymptotically stable if and only if $\forall i=1,2, \ldots, \omega$ and for $\phi(\lambda)=0$

$$
|\lambda|<1, \lambda \in \mathbb{C} .
$$

For the proof of (b), since governmental expenditure is a fully controlled variable for each country, equation (10) will take the form

$$
\begin{aligned}
T_{k}= & \bar{G}_{k}+\left[C^{(1)}+A^{(1)}+B A^{(1)}+\left(E-I_{\omega}\right) M^{(1)}\right] T_{k-1} \\
& +\sum_{q=2}^{p-1}\left\{\left[C^{(q)}+A^{(q)}+B\left(A^{(q)}-A^{(q-1)}\right)+\left(E-I_{\omega}\right) M^{(q)}\right] T_{k-q}\right\} \\
& +\left[C^{(p)}-B A^{(p-1)}+\left(E-I_{\omega}\right) M^{(p)}\right] T_{k-p}, \quad k \geq 0, \quad p \geq 3 .
\end{aligned}
$$

By adopting (14) and applying it into the above expression, we arrive at (13) which is a linear discrete-time control system with input vector $G_{k}$. Linear control involves modification of the behaviour of a given system by applying state feedback. It is known Azzo and Houpis (1995), Dorf (1983), Kuo (1996), Ogata (1987) that this is possible if and only if the system is completely controllable. The necessary and sufficient condition for complete controllability is that

$$
\operatorname{rank}\left[Q F Q F^{2} Q \cdots F^{p-1} Q\right]=p \omega .
$$

Since

$$
\operatorname{det}\left[Q F Q F^{2} Q \cdots F^{p-1} Q\right]=(-1)^{p \omega} \neq 0,
$$

which means system (13) is controllable and the state feedback replaces the input $G_{k}$ by

$$
\bar{G}_{k}=-K Y_{k}
$$

where

$$
K=\left[\begin{array}{llll}
K_{1} & K_{2} & \cdots & K_{p}
\end{array}\right]
$$

and $K_{1}, K_{2}, \ldots, K_{p} \in \mathbb{C}^{\omega \times \omega}$. The system then takes the form

$$
Y_{k+1}=(F-Q K) Y_{k},
$$

where $Q K=\left[\begin{array}{c}0_{\omega, \omega} \\ \vdots \\ 0_{\omega, \omega} \\ K\end{array}\right]$. The basic problem is that of choosing a state feedback $K$ such that the resulting (closed-loop equation) is stable. The stabilisation in the time-invariant case 
is via results on eigenvalue placement in the complex plane. In our situation, eigenvalues of the closed-loop system are specified to have modulus less than unity for stability. Thus, we have

$$
F-Q K=\left[\begin{array}{cccccc}
0_{\omega, \omega} & I_{\omega} & 0_{\omega, \omega} & \cdots & 0_{\omega, \omega} & 0_{\omega, \omega} \\
0_{\omega, \omega} & 0_{\omega, \omega} & I_{\omega} & \cdots & 0_{\omega, \omega} & 0_{\omega, \omega} \\
\vdots & \vdots & \vdots & \ddots & \vdots & \vdots \\
0_{\omega, \omega} & 0_{\omega, \omega} & 0_{\omega, \omega} & \cdots & 0_{\omega, \omega} & I_{\omega} \\
v_{1}-K_{1} & \nu_{2}-K_{2} & \nu_{3}-K_{3} & \cdots & v_{p-1}-K_{p-1} & v_{p}-K_{p}
\end{array}\right]
$$

where $v_{i}, i=1,2, \ldots, \omega$ are given by (14). Then, the characteristic equation of $F-Q K$ will be $\tilde{\phi}(\tilde{\lambda})$, with $\tilde{\lambda}$ the desired eigenvalues. The proof is completed.

\section{Numerical examples}

In this section, we present two numerical examples in order to provide further insight and better understanding regarding the system design and produced business cycles. For both examples, we model $\omega=5$ countries that each look back $p=3$ time steps. Let $A^{(1)}$, $A^{(2)}, B$ and $C^{(3)}$ take the following values:

\begin{tabular}{|c|c|c|c|c|c|}
\hline & 0.26 & 0.07 & 0.07 & 0.04 & 0.03 \\
\hline $\operatorname{iag}\{0.3,0.5,0.2,0.35,0.4\}$ & 0.39 & 0.30 & 0.27 & 0.21 & 0.10 \\
\hline $\operatorname{diag}\{0.3,0.1,0.25,0.25,0.35\}$ & 0.39 & 0.35 & 0.33 & 0.29 & 0.02 \\
\hline$=\operatorname{diag}\{0,5,07,0,6,0,4,0.3\}$ & 0.29 & 0.26 & 0.20 & 0.03 & 0.01 \\
\hline & 0.32 & 0.21 & 0.20 & 0.19 & 0.06 \\
\hline
\end{tabular}

while $\bar{E}$ and $M^{(i)}, i=1,2,3$, are given by:

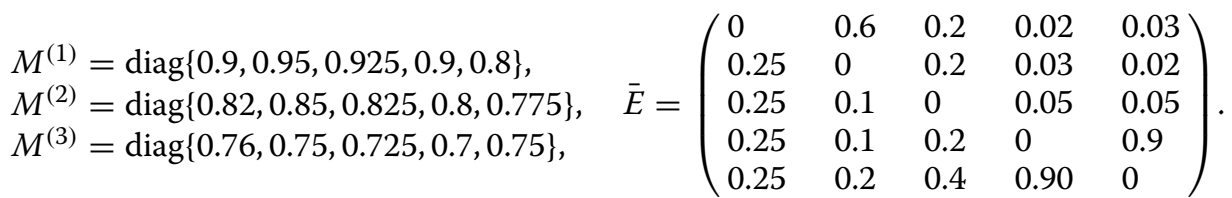

In addition let $C^{1}, C^{2}$ be given by:

$C^{(1)}=\left(\begin{array}{lllll}c_{1}^{1,1} & 0.32 & 0.28 & 0.24 & 0.20 \\ 0.32 & 0.28 & 0.24 & 0.20 & 0.16 \\ 0.28 & 0.24 & 0.20 & 0.12 & 0.08 \\ 0.24 & 0.20 & 0.12 & 0.08 & 0.04 \\ 0.20 & 0.12 & 0.08 & 0.04 & 0.01\end{array}\right), \quad C^{(2)}=\left(\begin{array}{ccccc}c_{2}^{1,1} & 0.24 & 0.10 & 0.04 & 0.01 \\ 0.36 & 0.30 & 0.26 & 0.25 & 0.13 \\ 0.34 & 0.27 & 0.20 & 0.20 & 0.13 \\ 0.32 & 0.31 & 0.28 & 0.19 & 0.05 \\ 0.36 & 0.29 & 0.29 & 0.23 & 0.08\end{array}\right)$.

In the matrices $C^{(1)}$ and $C^{(2)}$, the first elements (i.e. $c_{1}^{1,1}$ and $c_{2}^{1,1}$ ) are not assigned specific numerical values as these entries are varied in Examples 4.1 and 4.2.

\subsection{Example 4.1}

For this example, we assume that $c_{2}^{1,1}$ takes the value 0.36. Then, we may use Theorem 3.1 and determine the value of parameter $c_{1}^{1,1}$ such that the system is stable. Note that through $c_{1}^{1,1}$ the government of country $i=1$ controls the relevant governmental expenditure in order to obtain asymptotic stability in the system of national economy. The speed of the system's response is basically characterised by the maximum value $r$ of the following set 


$$
r=\max \{|\lambda|, \forall \lambda \in \mathbb{C}, \phi(\lambda)=0\}
$$

where $\lambda$ are the roots of (11). High-speed response coincides with minimising the value of $r$. According to Theorem 3.1, we require to have $r<1$, which is satisfied for $0<c_{1}^{1,1}<6.1$, see Figs. 1 and $2, c_{1}^{1,1}$.

\subsection{Example 4.2}

For this example, we may use Theorem 3.1 and determine the value of parameters $c_{1}^{1,1}$ and $c_{2}^{1,1}$ such that the system is stable. Note that through $c_{1}^{1,1}$ and $c_{2}^{1,1}$, the government of country $i=1$ controls the relevant governmental expenditure in order to obtain asymptotic stability in the system of national economy. The speed of the system's response is again characterised by the maximum value $r$, defined in Example 4.1. As before,

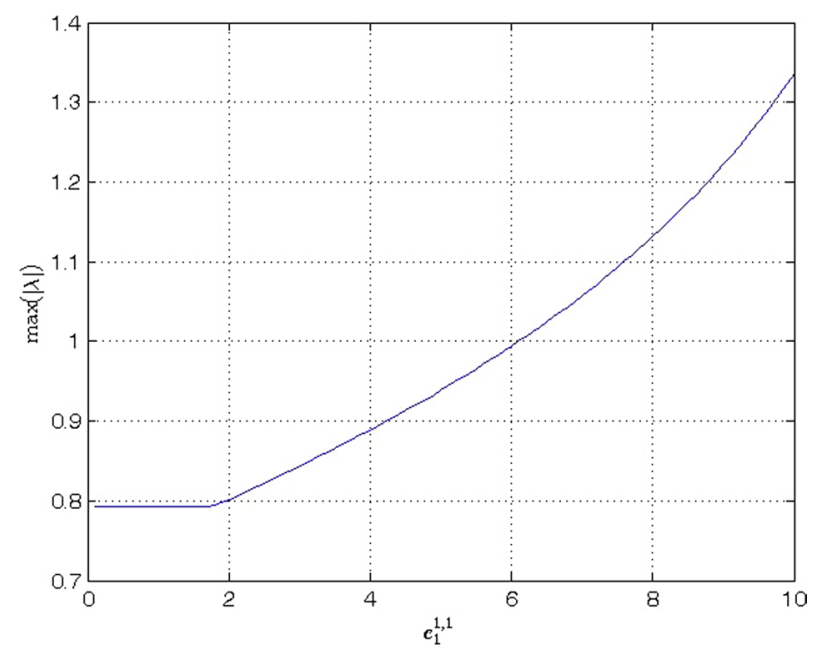

Fig. 1 Illustration of 2D diagram between $r$ and $c_{1}^{1,1}$

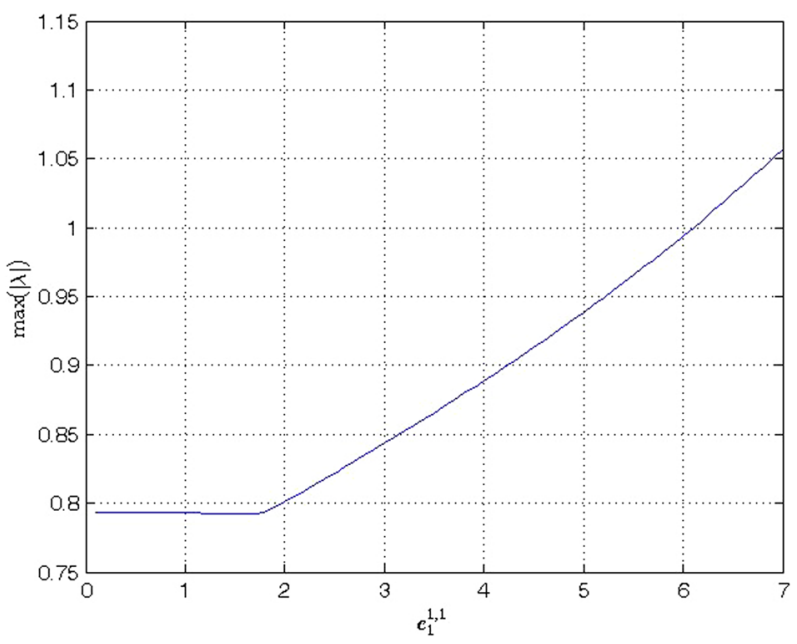

Fig. 2 Illustration of 2D diagram between $r$ and $c_{1}^{1,1}$ 
high-speed response coincides with minimising the value of $r$. Again, according to Theorem 3.1, we require to have $r<1$. Figures 3, 4 and 5 show the regions for which this is true. Figure 3 is a three-dimensional graph showing the values of $r$ for different values of $c_{1}^{1,1}$ and $c_{2}^{1,1}$. In Fig. 4, the different colours represent the different values of $r$. Figure 5 displays when the system is stable (blue region) and unstable (red region), i.e. when $r<1$ and $r \geq 1$, respectively.

\section{Discussion}

A further extension of this paper is to apply (incorporate) fractional operators into the model. The fractional nabla operator is a very interesting tool for this, since it succeeds to provide information from a specific year in the past until the current year, see Dassios and Baleanu (2013), Dassios et al. (2014), Dassios (2015)

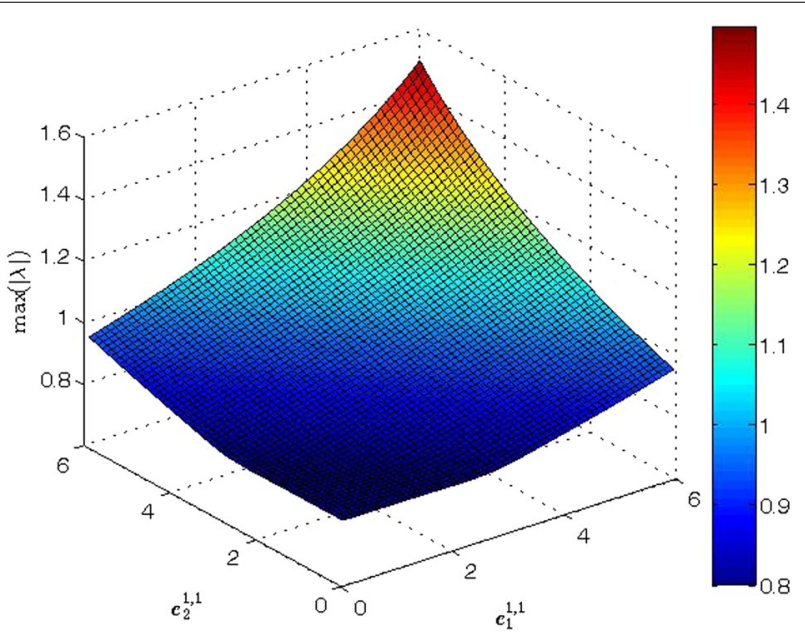

Fig. 3 Illustration of 3D diagram between $r, c_{1}^{1,1}$ and $c_{2}^{1,1}$

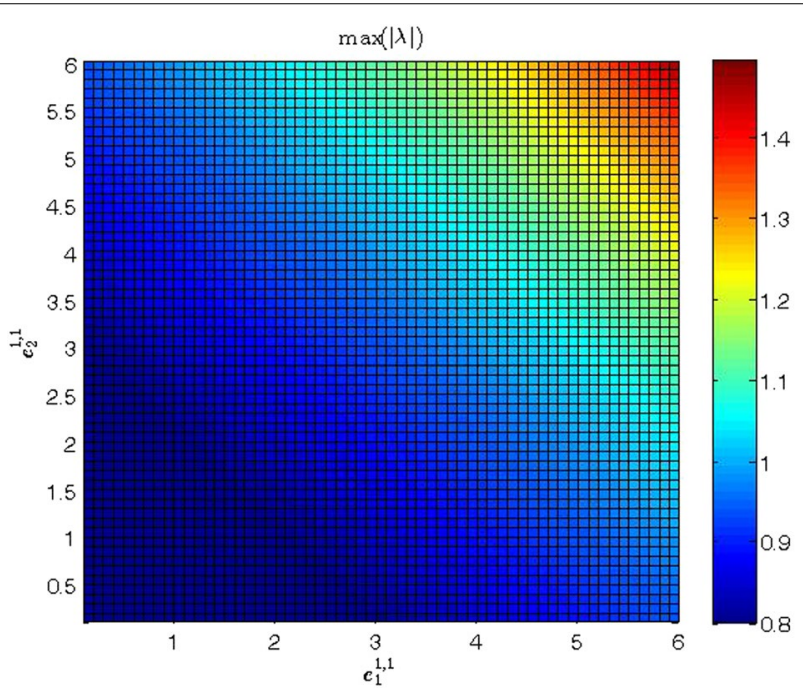

Fig. 4 Illustration diagram between $c_{1}^{1,1}$ and $c_{2}^{1,1}$. The bar on the right represents the values of $r$ 


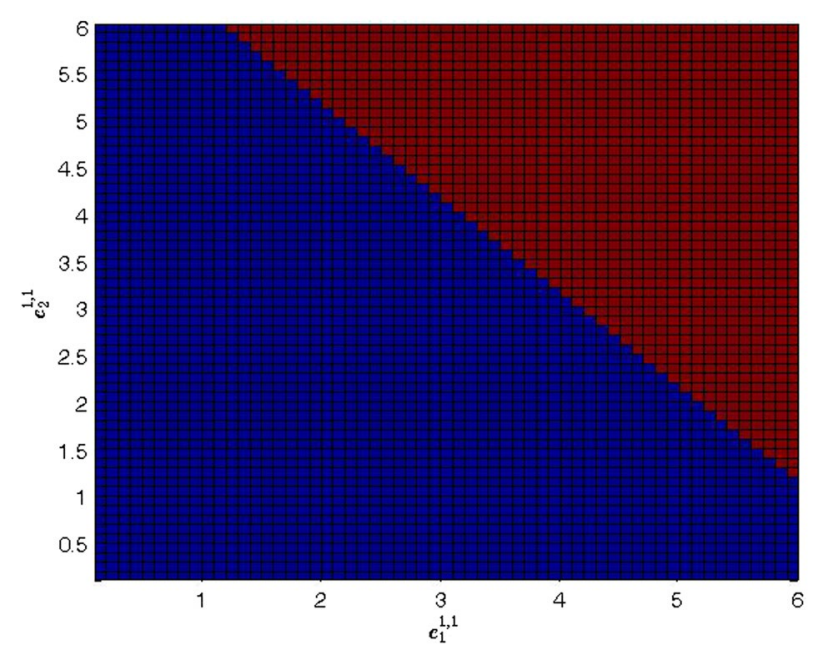

Fig. 5 Illustration diagram which displays when the system is stable (blue region) and unstable (red region)

- The nabla operator of $n$th order, $n$ Natural, applied to a vector of sequences $Y_{k}: \mathbb{N}_{\alpha} \rightarrow \mathbb{C}^{m}$ is defined by:

$$
\nabla^{n} Y_{k}=\sum_{j=0}^{n} a_{j} Y_{k-j}=a_{0} Y_{k}+a_{1} Y_{k-1}+\cdots+a_{n} Y_{k-n},
$$

where $a_{j}=(-1)^{j} \frac{1}{\Gamma(n+1) \Gamma(j+1) \Gamma(n-j+1)}$.

- The nabla fractional operator of $n$th order, $n$ Fractional, applied to a vector of sequences $Y_{k}: \mathbb{N}_{\alpha} \rightarrow \mathbb{C}^{m}$ is defined by

$$
\nabla_{\alpha}^{n} Y_{k}=\sum_{j=\alpha}^{k} b_{j} Y_{j}=b_{k} Y_{k}+b_{k-1} Y_{k-1}+\cdots+b_{\alpha} Y_{\alpha},
$$

where $b_{j}=\frac{1}{\Gamma(-n)}(k-j+1)^{\overline{-n-1}}$ and $(k-j+1)^{\overline{-n-1}}=\frac{\Gamma(k-j-n)}{\Gamma(k-j+1)}$.

Results on stability, robustness, duality, etc., of this operator, see Dassios (2016), Dassios and Baleanu (2015), Dassios (2015), may also be used successfully on the suggested macroeconomic models.

Furthermore, the coefficients used in the model (i.e. $a_{q}^{i}, b^{i}$ and $c_{q}^{i, d}$ ) are all deterministic and assumed exactly known. In reality, it may be difficult to determine these exactly, particularly coefficients that represent predicted future values. Future work will examine the effects and stability of making the coefficients in the model stochastic. For all these, there is some research in progress.

\section{Conclusions}

In this article, we assumed a union of countries where each national economy interacts with the others according to past year's experience and trade relations (exportsimports). We considered the equilibrium of this model (a discrete-time system) and provided a theorem for its stability, the appropriate control actions and the total system design in order to obtain a stable situation. Finally, numerical examples were given in 
order to provide further insight and better understanding as regards the system design and produced business cycles.

\author{
Author details \\ ${ }^{1}$ Mathematics Applications Consortium for Science and Industry (MACSI), Department of Mathematics and Statistics, \\ University of Limerick, Limerick, Ireland. ${ }^{2}$ Economic and Social Research Institute (ESRI), Whitaker Square, Dublin 2, \\ Ireland. ${ }^{3}$ Department of Economics, Trinity College Dublin, Dublin, Ireland.
}

\title{
Acknowledgements
}

Both authors acknowledge Science Foundation Ireland award 09/SRC/E1780, while M. T. Devine also acknowledges the ESRI's Energy Policy Research Centre.

\section{Competing interests}

The authors declare that they have no competing interests.

Received: 15 December 2015 Accepted: 7 May 2016

Published online: 08 June 2016

\section{References}

Azzo JD, Houpis CH (1995) Linear control system analysis and design, 4th edn. Mc Graw-Hill, New York

Chari VV (1994) Optimal fiscal policy in a business cycle model. J Polit Econ 102(4):52-61

Chow GC (1985) A model of Chinese national income determination. J Polit Econ 93(4):782-792

Dalla E, Varelas E (2015) Second-order accelerator of investment: the case of discrete time. Int Rev Econ Educ 21:48-60

Dalla E, Karpetis C, Varelas E (2016) Modeling investment cycles: a theoretical analysis. Mod Econ 7(03):336

Dassios I, Zimbidis A, Kontzalis C (2014) The delay effect in a stochastic multiplier-accelerator model. J Econ Struct 3(1):7

Dassios I, Kalogeropoulos G (2014) On the stability of equilibrium for a reformulated foreign trade model of three countries. J Ind Eng Int 10:71. doi:10.1007/s40092-014-0071-9

Dassios I, Zimbidis A (2014) The classical Samuelson's model in a multi-country context under a delayed framework with interaction. Dyn Contin Discrete Impuls Syst Ser B Appl Algorithms 21(4-5b):261-274

Dassios IK, Baleanu D (2013) On a singular system of fractional nabla difference equations with boundary conditions. Bound Value Probl 2013:148

Dassios I, Baleanu D, Kalogeropoulos G (2014) On non-homogeneous singular systems of fractional nabla difference equations. Appl Math Comput 227:112-131

Dassios I (2016) Stability and robustness of singular systems of fractional nabla difference equations. Circuits Syst Signal Process. doi:10.1007/s00034-016-0291-x

Dassios I, Baleanu D (2015) Duality of singular linear systems of fractional nabla difference equations. Appl Math Model 39(14):4180-4195

Dassios IK (2015) Optimal solutions for non-consistent singular linear systems of fractional nabla difference equations. Circuits Syst Signal Process 34(6):1769-1797

Dassios I (2015) Geometric relation between two different types of initial conditions of singular systems of fractional nabla difference equations. Methods Appl Sci Math. doi:10.1002/mma.3771

Day R (1999) Complex economic dynamics: an introduction to macroeconomic dynamics, vol 2. MIT Press, Cambridge

Dorf RC (1983) Modern control systems, 3rd edn. Addison-Wesley, Boston

Karpetis C, Varelas E (2012) Fiscal and monetary policy interaction in a simple accelerator model. Int Adv Econ Res 18(2):199-214

Kotsios S, Leventidis J (2004) A feedback policy for a modified Samuelson-Hicks model. Int J Syst Sci 35(6):331-341

Kotsios I, Kostarakos S (2015) Fiscal policy design in Greece in the aftermath of the crisis: an algorithmic approach. https://www.researchgate.net/

Kotsios I, Kostarakos S (2016) Feedback policy rules for government spending: an algorithmic approach. https://www. researchgate.net/

Kuo BC (1996) Automatic control systems, 5th edn. Prentice Hall, Upper Saddle River

Machado JA, Mata ME, Lopes AM (2015) Fractional state space analysis of economic systems. Entropy 17(8):5402-5421

Matsumoto A, Szidarovszky F (2015) Nonlinear multiplier? Accelerator model with investment and consumption delays. Struct Change Econ Dyn 33(2015):1-9

Ogata K (1987) Discrete time control systems. Prentice Hall, Upper Saddle River

Puu T, Gardini L, Sushko I (2004) A Hicksian multiplier-accelerator model with floor determined by capital stock. J Econ Behav Org 56(3):331-348

Rosser JB (2000) From catastrophe to chaos: a general theory of economic discontinuities. Academic Publishers, Boston

Samuelson P (1939) Interactions between the multiplier analysis and the principle of acceleration. Rev Econ Stat 21(2):75-78

Westerhoff FH (2006) Samuelson's multiplier-accelerator model revisited. Appl Econ Lett 56:86-92

Wincoop E (1996) A multi-country real business cycle model. Scand J Econ 23:233-251 